\title{
Modelo de inferencia borroso para el cálculo de indicadores claves de rendimiento
}

\author{
Fuzzy inference model for the calculation of key performance indicators \\ Francisco J. Peña Veitía ${ }^{1 *} \quad$ Elieser E. Bello $^{1} \quad$ Rafael Bello $^{2}$ \\ Gheisa L. Ferreira Lorenzo ${ }^{2,3}$ Ana M. García Pérez ${ }^{1}$ \\ Recibido 20 de enero de 2019, aceptado 10 de marzo de 2020 \\ Received: January 20, 2019 Accepted: March 10, 2020
}

\begin{abstract}
RESUMEN
Las aplicaciones de inteligencia de negocio utilizan de manera más frecuente técnicas de inteligencia artificial para realizar predicciones, manejar la incertidumbre e incidir en la toma de decisiones organizacionales. El sistema ejecutivo para el seguimiento a estrategias organizacionales - BIMAS, es una solución informática para la gestión del desempeño e inteligencia empresarial, desarrollada y comercializada por la empresa cubana DESOFT que evalúa los indicadores claves de rendimiento de la empresa de manera rígida a través de rangos definidos por los usuarios. Esta evaluación de indicadores en el tiempo ha presentado resultados que en ocasiones no se ajustan a la realidad; al ser una evaluación dirigida por el usuario, no se han considerado intervalos de tiempo para la misma y lo que para algunos, un indicador puede resultar "bajo", para otros pudiera considerarse "medio" e incluso "alto". De ahí que el objetivo de este trabajo radique en implementar un modelo de inferencia borroso que tome en consideración aspectos objetivos y subjetivos que caracterizan el desempeño de los procesos en las organizaciones, eligiendo en este caso como términos para el análisis, el porciento de cumplimiento y el período de evaluación de cada indicador. Se pretende demostrar que con este modelo se obtienen evaluaciones flexibles y cercanas a la realidad, brindando a los decisores información confiable respecto a sus organizaciones.
\end{abstract}

Palabras clave: Lógica difusa, indicadores claves de rendimiento, sistema de inferencia borroso.

\begin{abstract}
Business intelligence applications use artificial intelligence techniques more frequently to make predictions, handle uncertainty, and influence organizational decision making. The executive system for monitoring organizational strategies - BIMAS, is a computer solution for performance management and business intelligence, developed and marketed by the Cuban company DESOFT that evaluates the company's key performance indicators rigorously through ranges defined by users. This evaluation of indicators over time has presented results that sometimes do not conform to reality; being a user-directed evaluation, time intervals have not been considered for it, and what for some, an indicator may be "low", for others it could be considered "medium" and even "high." Hence, this work aims to implement a fuzzy inference model that considers objective and subjective aspects that characterize the performance of processes in organizations, choosing in this case as terms for the analysis of the percentage of compliance and Evaluation period of each indicator. It is intended to demonstrate that flexible and close to reality evaluations are obtained with this model, providing decision-makers with reliable information regarding their organizations.
\end{abstract}

Keywords: Fuzzy logic, Key performance indicators, fuzzy inference system.

\footnotetext{
1 Empresa de desarrollo de software DESOFT. Villa Clara, Cuba.

E-mail: francisco.pena@desoft.cu; elieser.bello@desoft.cu; anamaria.garcia@desoft.cu

2 Centro de Investigaciones de la Informática. Universidad Central "Marta Abreu" de Las Villas". Villa Clara, Cuba.

E-mail: rbellop@uclv.edu.cu; gheisa@uclv.edu.cu

3 Departamento Ingeniería de Sistemas. Universidad Metropolitana del Ecuador. Quito, Ecuador. E-mail: gferreira@umet.edu.ec

* Autor de correspondencia: fjpena35226@gmail.com
} 


\section{INTRODUCCIÓN}

En la actualidad, la dinámica de los negocios está avanzando rápidamente, en la misma medida que va evolucionando la necesidad técnica de adaptarse a las exigencias de un mercado complejo y en constante cambio. Esto requiere de metodologías y herramientas que aseguren el éxito a mediano y largo plazo. El Cuadro de Mando Integral (CMI) o Balance Scorecard desarrollado por [1] es una herramienta utilizada en la Inteligencia de Negocios [2]. Dentro del CMI, los indicadores claves de rendimiento (KPI, Key Perfomance Indicators, por sus siglas en inglés); son un aspecto importante a tener en cuenta en el desarrollo de cualquier modelo de negocio actual. El CMI como herramienta de control de gestión, es un método de medición del rendimiento que integra indicadores financieros y no financieros derivados de la estrategia organizacional y ayuda a los directivos en la toma de decisiones a través de los KPI, para saber si se alcanzan los objetivos estratégicos y con ello el éxito de la empresa.

Las soluciones informáticas de inteligencia de negocio cada día se vuelven más "inteligentes" al utilizar técnicas y algoritmos de inteligencia artificial para el mejor entendimiento de las decisiones en los negocios y la predicción de qué podría pasar en el futuro. Se hace necesario el empleo de las técnicas de inteligentes teniendo en cuenta que los procesos de toma de decisiones más complejos son típicamente no estructurados, es decir, no existen reglas o procedimientos que determinen qué decisión tomar en el caso de la existencia de diferentes alternativas [3]. Como parte de la inteligencia artificial y las matemáticas, y buscando una mayor flexibilidad y naturalidad para manejar la incertidumbre inherente a los dominios de aplicación, la lógica difusa [4] es utilizada para construir modelos para la toma de decisiones, entre ellos los Sistemas de Inferencia Borrosa (SIB) [5, 6]. Tal es el caso que se menciona en [7] donde se toma como eje del modelo, las relaciones entre los objetivos estratégicos. Ideas similares aparecen en [8, 9 y 10]. Sin embargo, en los casos mencionados anteriormente no se toma en consideración el período en que se está evaluando el indicador. Este es un punto clave a tener en cuenta, ya que un mismo indicador puede tener distintas consideraciones y evaluaciones dependiendo del período en que se debe evaluar; es decir, un indicador al cuál se le hace una planificación a cumplir en un período de 30 días puede tener distintas evaluaciones en las fases de inicio, medio y fin.

La empresa cubana de software DESOFT desarrolla y comercializa el sistema ejecutivo para el seguimiento a estrategias organizacionales - BIMAS [11], que en su versión actual está formado por cuatro componentes: BIMAS Smifor, BIMAS Bion Windows, BIMAS Bios Android y BIMAS Server. El componente BIMAS Smifor es un sistema distribuido que extrae los datos de los diferentes sistemas de gestión, y envía el resultado como una tabla de datos hacia BIMAS Server, en un formato .dbf, .xls o .csv, por un canal email, ftp, http y con una frecuencia determinada. BIMAS Bion Windows y Android son aplicaciones de escritorio o para dispositivos móviles, respectivamente, que visualizan en tiempo real el valor de indicadores que el usuario seleccione y su comportamiento. Por su parte BIMAS Server, es el núcleo de la aplicación y permite la configuración, planificación y ejecución estratégica a organizaciones y sus estructuras, así como variadas aplicaciones colaborativas como calendarios corporativos e individuales, foros de discusión, encuestas, entre otros. El presente trabajo se centra en BIMAS Server. En este componente, los indicadores claves de rendimiento se evalúan de manera rígida a través de escalas definidas por los usuarios. Por ejemplo, un indicador se considera evaluado de "mal" si se encuentra en un porciento de cumplimiento entre $0 \%$ y $70 \%$, "regular" si se encuentra entre un $71 \%$ y un $95 \%$ y "bien" si se encuentra entre un $96 \%$ y un $100 \%$; obteniéndose resultados en múltiples casos alejados a la realidad, al no considerar el momento en el tiempo al realizarse la evaluación. En lugar de obtener una evaluación ya prefijada en una escala, el objetivo de este trabajo consiste en implementar un modelo de inferencia borroso que tome en consideración algunos indicadores claves de rendimiento, para evaluarlos utilizando valores cualitativos de "bien", "regular" y "mal", modelados como variables lingüísticas en un sistema de inferencia borroso. En este caso se eligen el porciento de cumplimiento y el período de evaluación de cada indicador por estar estrechamente relacionados. Se pretende demostrar que con este modelo se obtienen evaluaciones flexibles y cercanas a la realidad, lo que facilitará información al decisor con la que podrá evaluar los objetivos estratégicos y perspectivas de su organización. 


\section{DESARROLLO}

\section{Lógica difusa y sistemas de inferencia borrosos} En el desarrollo de la investigación fueron analizados algunos conceptos básicos de la lógica difusa. Estos conceptos parten de que cotidianamente se utiliza en el lenguaje, la idea de las clases de conjuntos que comparadas con los conjuntos matemáticos dejan margen a la incertidumbre. Por ejemplo, cuando se califica el "porciento de cumplimiento" de "bajo" o "alto" ¿cuáles son los intervalos a considerar? Es posible que, para algunas personas, el calificativo de "bajo" se encuentre entre ciertos valores, mientras que para otras se encuentre acotado en otro intervalo; por tanto, este tipo de calificativos se encuentran sujetos a la opinión subjetiva de cada persona y al contexto donde se desee evaluar la situación. Lo mismo ocurre con la evaluación del desempeño de una persona en su trabajo, que pudiera considerarse de "regular" por algunos evaluadores, mientras que por otros pudiera evaluarse de "bien".

Estos ejemplos sirven para ilustrar el hecho de que la transición de un conjunto $\mathrm{X}$ a un conjunto $\mathrm{X}$ ' es continua en el mundo físico, en lugar de presentar límites visibles; las fronteras son vagas y subjetivas. En 1965, basado en el concepto fundamental de variación de temperatura o multivalencia, el profesor Lotfi A. Zadeh del Departamento de Ingeniería Eléctrica de la Universidad de California en Berkley formalizó la teoría de los conjuntos difusos mediante la publicación del artículo [12].

La teoría ha sido utilizada en múltiples escenarios. En el análisis de las finanzas y recursos humanos en las empresas [13,14], se proponen marcos de trabajo para el análisis de una inserción exitosa de la empresa en el mercado internacional (a través de un conjunto amplio de variables estratégicas como: talento humano, infraestructura, estrategias de innovación, acuerdos de libre comercio, finanzas y estrategias de marketing), especificándose en los resultados los factores y variables sobre los que intervenir para mejorar la capacidad de exportación. De igual manera se perfeccionan los estados financieros dispuestos en normativas vigentes, con la adición de estados financieros previsionales que repercuten positivamente en la disminución de la incertidumbre y la subjetividad que caracteriza a la dinámica empresarial. Con relación a la responsabilidad social y el ambiente laboral $[15,16]$ resulta interesante destacar cómo se parametrizan los indicadores de desempeño organizacional en cuanto a sostenibilidad, marco legal y desempeño anual en un instrumento efectivo para la evaluación de este enfoque de gestión. En la medicina, tal como aparece en [17], aparecen soluciones para tratamientos de enfermedades que mezclan la lógica borrosa con otras técnicas de inteligencia artificial como los algoritmos genéticos. Se hace notar cómo, a diferencia de los trabajos anteriores donde las soluciones se modelaban con funciones triangulares, en este caso se combinan en varias iteraciones funciones triangulares y gaussianas para mejorar la solución. También se ha aplicado la lógica borrosa en la conceptualización y evaluación de la calidad de los procesos de negocio [18, 19], con la definición de reglas borrosas a partir de guías y buenas prácticas utilizando un estándar para el modelado. De manera general la lógica difusa aparece como una herramienta que permite modelar modos de razonamiento imprecisos, que son importantes para la toma de decisiones racionales en un intervalo de incertidumbre e imprecisión.

Para atender este modelado, se define el sistema de inferencia borroso, como un mecanismo que permite modelar los conjuntos donde las fronteras son vagas, a partir de funciones no lineales. Este sistema tiene variables lingüísticas de entrada, que convierte en variables de salida mediante la lógica difusa y un conjunto de reglas, según se muestra en la Figura 1.

El proceso que se muestra en la Figura 1, se puede resumir en los siguientes pasos:

1. Interpretar los valores de entrada rígidos o borrosos a través de las funciones de pertenencia de las variables lingüísticas de entrada, usualmente llamado "fusificación".

2. Asignar valores al vector de salida, basado en un conjunto de reglas de control borrosas, a través de un mecanismo de inferencia.

3. Interpretar el vector de salida por las variables lingüísticas de salida, con un método de "defusificación", dando como resultado valores rígidos.

La formulación, de manera general involucra:

- Diseño de las variables lingüísticas, que están dadas según [12] por $<\mathbf{N}, \mathbf{U}, \mathbf{T}(\mathbf{N}), \mathbf{M}>$; donde: $-\mathbf{N}$ es el nombre de la variable lingüística. $-\mathbf{U}$ es el dominio subyacente. 


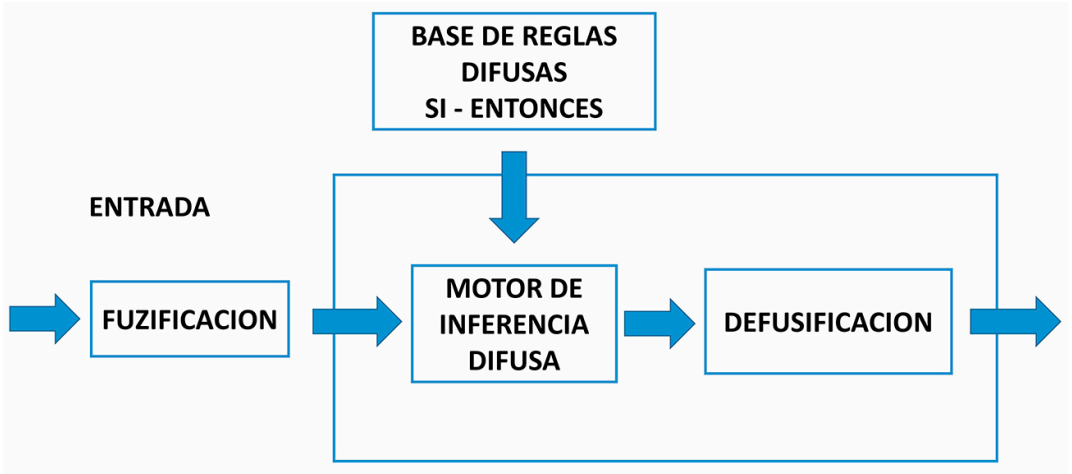

Figura 1. Sistema de Inferencia Borroso.

- T(N) es el conjunto de términos o etiquetas lingüísticas que puede tomar $\mathbf{N}$ y $\mathbf{M}$; es una regla que asocia cada elemento de $\mathbf{T}(\mathbf{N})$ con un conjunto difuso en $\mathbf{U}$ de entre todos los posibles.

Ejemplo: <porciento_cumplimiento, [0;1], $\{$ MUY_BAJO, BAJO, MEDIO, ALTO $\}$

- Diseño de las funciones de pertenencia, que pueden ser representadas como $\mathbf{A}: \mathbf{X} \rightarrow[\mathbf{0 ;} \mathbf{1}]$ describiendo un conjunto borroso que mapea entradas rígidas hasta su grado de pertenencia. Estas, en dependencia de la variable que representen, pueden ser de tipo trapezoidal, singleton, triangular, $\mathrm{S}$, exponencial, entre otras [20].

- Diseño de las reglas borrosas, como formas de representar el conocimiento consciente de uno o varios expertos, escritas del tipo SI-ENTONCES, respecto a algún problema determinado.

Ejemplo:

SI porciento_cumplimiento es MEDIO y tiempo_ejecución es INICIO ENTONCES evaluación es BIEN

- Finalmente, el método de "defusificación" para la obtención de la salida del sistema. Existen varios métodos de "defusificación". De ellos, los más utilizados son el centro de gravedad y la duración media máxima. Con el centro de gravedad, la salida es el valor en el universo que separa el área bajo la curva de la función en dos partes iguales.

Un vez establecido el modelo de inferencia borroso, el Lenguaje de Control Borroso (Fuzzy
Control Language, FCL por sus siglas en inglés) permite su implementación. FCL es un estándar para la programación de lógica borrosa de control publicado por la International Electrotechnical Commission en la norma 61131-7 [8]. FCL ofrece una comprensión común de términos básicos de integración de aplicaciones de control borroso en sistemas de control, proporcionando un lenguaje común con el que implementar programas de control difuso portátiles entre diferentes plataformas. Tiene como principales ventajas la fácil migración entre distintas plataformas de hardware-software, de diferentes fabricantes y la reutilización de la aplicación desarrollada.

En los últimos años se han desarrollado herramientas para el trabajo con la lógica difusa. De estas, solo pocas de código abierto [21]. Para la implementación de los resultados de esta investigación se ha utilizado la biblioteca de código abierto jFuzzyLogic, que permite diseñar e implementar un sistema de inferencia borroso con este estándar. Dentro de sus ventajas se encuentran:

- Estandarización del código reduciendo la necesidad de los investigadores de tener conocimientos profundos en lógica borrosa de control.

- Enfoque orientado a objetos sobre la que se encuentra desarrollada la biblioteca, que permite una fácil extensión de la misma y su uso en cualquier configuración de un sistema que soporte Java debido al enfoque independiente de plataforma.

Con el uso de jFuzzyLogic, en este trabajo se implementó un controlador en Java para el SIB diseñado que es capaz de evaluar los KPI, 
siguiendo técnicas de programación que facilitan su extensibilidad y modularidad.

\section{RESULTADOS Y DISCUSIÓN}

Para la construcción del SIB en este problema, se procede de acuerdo a los pasos establecidos para el mismo en el epígrafe anterior.

En la evaluación de los indicadores claves de rendimiento en la empresa, existen dos variables lingüísticas que son importantes en el momento de emitir una evaluación. Estas variables son:

- Porciento de cumplimiento: describe, respecto a un plan de cumplimiento, qué porciento de ese indicador se ha logrado en términos de MUY_BAJO, BAJO, MEDIO y ALTO.

- Tiempo de ejecución: describe, para un período de evaluación acotado en una fecha de inicio y una fecha de fin, en qué momento de ese período se está evaluando el indicador en términos de INICIO, MEDIO y FIN.

Variables lingüísticas de entrada:

<PORCIENTO_CUMPLIMIENTO, [0;1], $\{$ MUY_BAJO, BAJO, MEDIO, ALTO $\}$
<TIEMPO_EJECUCION, [0;T], \{ INICIO, MEDIO, FIN $\}>$ donde $\mathbf{T}$ es la cantidad en días del período analizado.

La relación entre estas dos variables puede dar una evaluación de cómo se encuentra el KPI, formulando la variable de salida Evaluación para que tome los valores de BIEN, REGULAR o MAL.

Variable lingüística de salida:

$<$ EVALUACIÓN, $\{1,2,3\}$, \{BIEN, REGULAR, MAL $>$

Para la construcción de las funciones de pertenencia fue beneficiosa la información y opinión subjetiva recopilada de entrevistas con los expertos. Esto permitió definir las funciones de pertenencia, de tipo trapezoidal, de cada una de las variables lingüísticas.

Las Figuras 2 y 3, representan las funciones de pertenencia para cada variable: PORCIENTO_ CUMPLIMIENTO y TIEMPO_EJECUCIÓN.

En la Figura 2, aparece representada la función de pertenencia de la variable PORCIENTO_ CUMPLIMIENTO. Esta función se define a partir

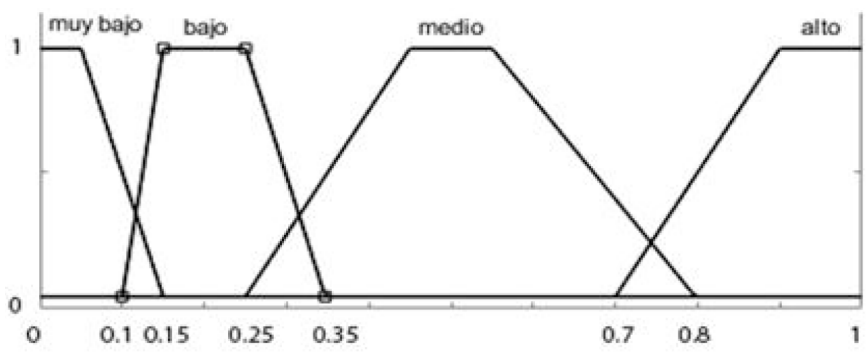

Figura 2. Función de pertenencia variable PORCIENTO_ CUMPLIMIENTO.

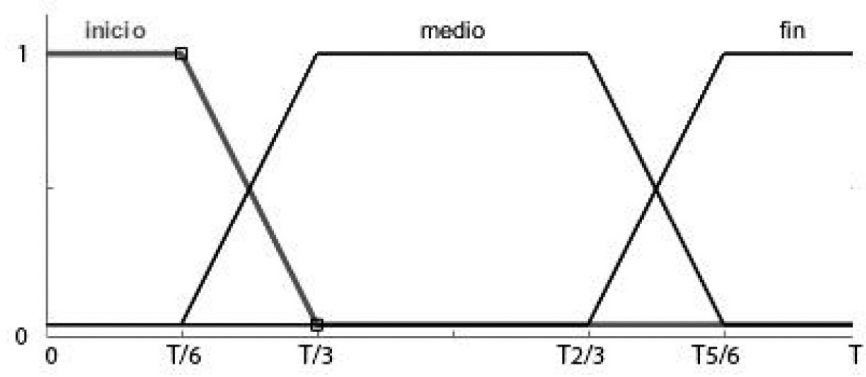

Figura 3. Función de pertenencia variableTIEMPO_EJECUCIÓN. 
de cuatro funciones no lineales trapezoidales. Los valores definidos por intervalos son resumidos en la Tabla 1.

La Figura 3 representa la función de pertenencia para la variable TIEMPO_EJECUCIÓN. El dominio subyacente se ha analizado en tercios, para establecer los intervalos que ocupa cada término lingüístico y que han sido representados como conjuntos en la Tabla 1.

La Tabla 1 resume los intervalos de análisis de cada función de pertenencia tratados anteriormente.

Suponiendo que la planificación realizada para cada indicador es aceptable, y de acuerdo con los estudios de comportamiento de dichas variables linguiísticas, se logró una generalización parcial para los indicadores que cuentan con un plan de cumplimiento, donde se pudo observar que:

1. Mientras más cercanos estén los valores de porciento de cumplimiento y tiempo de ejecución, mejor será la evaluación del indicador.

2. Mientras el valor de porciento de cumplimiento sea mejor que el del tiempo de ejecución, la evaluación del indicador será favorable.

3. Por el contrario, si el valor del porciento de cumplimiento es peor que el del tiempo de ejecución, la evaluación del indicador será desfavorable.

4. Debido a la variada naturaleza del comportamiento de los indicadores al exceder la planificación, o sea al sobrepasar el 100\%, se decide acotar en esta fase de la investigación el valor del porciento de cumplimiento al $100 \%$, de modo que si algún indicador sobrepasa el $100 \%$ será evaluado como si tuviera un $100 \%$ de cumplimiento.
Siguiendo la descripción y el comportamiento anterior se definen las siguientes reglas borrosas en la forma SI-ENTONCES:

1. SI porciento_cumplimiento es BAJO y tiempo_ ejecución es INICIO ENTONCES evaluación es BIEN.

2. SI porciento_cumplimiento es BAJO y tiempo_ ejecución es MEDIO ENTONCES evaluación es MAL.

3. SI porciento_cumplimiento es BAJO y tiempo_ ejecución es FIN ENTONCES evaluación es MAL.

4. SI porciento_cumplimiento es MEDIO y tiempo_ejecución es INICIO ENTONCES evaluación es BIEN.

5. SI porciento_cumplimiento es MEDIO y tiempo_ejecución es MEDIO ENTONCES evaluación es REGULAR.

6. SI porciento_cumplimiento es MEDIO y tiempo_ ejecución es FIN ENTONCES evaluación es MAL.

7. SI porciento_cumplimiento es ALTO y tiempo_ ejecución es INICIO ENTONCES evaluación es BIEN.

8. SI porciento_cumplimiento es ALTO y tiempo_ ejecución es MEDIO ENTONCES evaluación es BIEN.

9. SI porciento_cumplimiento es ALTO y tiempo_ ejecución es FIN ENTONCES evaluación es BIEN.

10. SI porciento_cumplimiento es MUY_BAJO y tiempo_ejecución es FIN ENTONCES evaluación es BIEN.

11. SI porciento_cumplimiento es MUY_BAJO y tiempo_ejecución es FIN ENTONCES evaluación es REGULAR.

12. SI porciento_cumplimiento es MUY_BAJO y tiempo_ejecución es FIN ENTONCES evaluación es MAL.

Tabla 1. Funciones de pertenencia.

\begin{tabular}{|l|c|c|c|c|}
\hline \multicolumn{1}{|c|}{ Variables } & \multicolumn{4}{|c|}{ Etiquetas lingüísticas } \\
\hline \multirow{2}{*}{ PORCIENTO } & MUY_BAJO & BAJO & MEDIO & ALTO \\
_CUMPLIMIENTO & $\{(0 ; 0)(0 ; 1)$ & $\{(0.1 ; 0)(0.15 ; 1)$ & $\{(0.25 ; 0)(0.45 ; 1)$ & $\{(0.7 ; 0)(0.9 ; 1)$ \\
& $(0.05 ; 1)(0.15 ; 0)\}$ & $(0.25 ; 1)(0.3 ; 0)\}$ & $(0.55 ; 1)(0.8 ; 0)\}$ & $(1 ; 1)(0 ; 1)\}$ \\
\hline \multirow{2}{*}{ TIEMPO } & & INICIO & MEDIO & FIN \\
_EJECUCION & & $\{(0 ; 0)(0 ; 1)(\mathrm{T} / 6 ; 1)$ & $\{(\mathrm{T} / 6 ; 0)(\mathrm{T} / 3 ; 1)$ & $\{(\mathrm{T} 2 / 3 ; 0)(\mathrm{T} 5 / 6 ; 1)$ \\
& & $(\mathrm{T} / 3 ; 0)\}$ & $(\mathrm{T} 2 / 3 ; 1)(\mathrm{T} 5 / 6 ; 0)\}$ & $(1 ; 1)(1 ; 0)\}$ \\
\hline \multirow{2}{*}{ EVALUACIÓN } & & BIEN & REGULAR & MAL \\
& & $\{(3 ; 0)\}$ & $\{(2 ; 0)\}$ & $\{(1 ; 0)\}$ \\
\hline
\end{tabular}


Posteriormente se implementa el Sistema de Inferencia Borroso. La capa controladora es implementada en Java utilizando la biblioteca jFUzzyLogic, como se puede apreciar en un fragmento de código que aparece en la Figura 4.

Se diseñó un experimento para probar la efectividad del modelo propuesto. Para el mismo se seleccionaron 120 registros (del registro histórico de la herramienta BIMAS con valores reales del indicador Ingresos por Servicios y su porciento de cumplimiento) de los meses de marzo, abril, mayo y junio de 2017. Adicionalmente, se realizó la evaluación de porciento de cumplimiento por un grupo de expertos que constituyen usuarios avanzados en el uso de la herramienta y finalmente por el sistema de inferencia borroso propuesto (IBIMAS). En la Tabla 2 aparece un fragmento del comportamiento de los resultados. Note que, en los resultados marcados en color rojo, no existe una total concordancia.

A fin de comprobar la validez de los resultados obtenidos con la propuesta de modelo de inferencia borroso, se aplicó una prueba de Kruskal-Wallis con comparaciones múltiples por pares mediante el procedimiento de Steel-Dwass-Critchlow-Fligner (prueba bilateral) que aportó los resultados que aparecen en la Tabla 3. Puede observarse que en este análisis la muestra se divide en dos grupos bien diferenciados: Grupo A (resultados de BIMAS), Grupo B (resultados de la evaluación por EXPERTO e IBIMAS).

Otro de los resultados observables de la prueba estadística es el que se muestra en la Tabla 4.

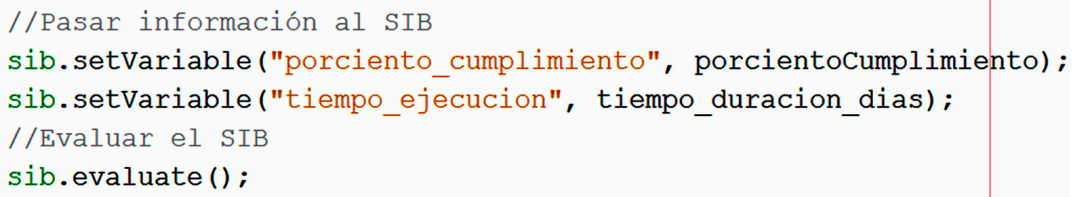

Figura 4. Implementación del controlador en Java.

Tabla 2. Resultados de la evaluación del indicador Ingresos por Servicios.

\begin{tabular}{|c|c|l|c|c|c|c|}
\hline Mes & Fecha & Indicador & \% cumplimiento & BIMAS & EXPERTO & IBIMAS \\
\hline marzo & $1 / 3 / 2017$ & Ingresos por Servicios & 0,00 & MAL & MAL & MAL \\
\hline marzo & $2 / 3 / 2017$ & Ingresos por Servicios & 0,00 & MAL & MAL & MAL \\
\hline marzo & $3 / 3 / 2017$ & Ingresos por Servicios & 0,00 & MAL & MAL & MAL \\
\hline marzo & $4 / 3 / 2017$ & Ingresos por Servicios & 0,00 & MAL & MAL & MAL \\
\hline marzo & $5 / 3 / 2017$ & Ingresos por Servicios & 0,00 & MAL & MAL & MAL \\
\hline marzo & $6 / 3 / 2017$ & Ingresos por Servicios & 0,00 & MAL & MAL & MAL \\
\hline marzo & $7 / 3 / 2017$ & Ingresos por Servicios & 0,00 & MAL & MAL & MAL \\
\hline marzo & $8 / 3 / 2017$ & Ingresos por Servicios & 0,00 & MAL & MAL & MAL \\
\hline marzo & $9 / 3 / 2017$ & Ingresos por Servicios & 0,00 & MAL & MAL & MAL \\
\hline marzo & $10 / 3 / 2017$ & Ingresos por Servicios & 7,33 & MAL & MAL & MAL \\
\hline marzo & $11 / 3 / 2017$ & Ingresos por Servicios & 7,33 & MAL & MAL & MAL \\
\hline marzo & $12 / 3 / 2017$ & Ingresos por Servicios & 7,33 & MAL & MAL & MAL \\
\hline marzo & $13 / 3 / 2017$ & Ingresos por Servicios & 35,16 & MAL & MAL & REGULAR \\
\hline marzo & $14 / 3 / 2017$ & Ingresos por Servicios & 35,16 & MAL & MAL & REGULAR \\
\hline marzo & $15 / 3 / 2017$ & Ingresos por Servicios & 72,50 & REGULAR & BIEN & REGULAR \\
\hline marzo & $16 / 3 / 2017$ & Ingresos por Servicios & 81,66 & REGULAR & BIEN & BIEN \\
\hline marzo & $17 / 3 / 2017$ & Ingresos por Servicios & 85,62 & REGULAR & BIEN & BIEN \\
\hline marzo & $18 / 3 / 2017$ & Ingresos por Servicios & 85,62 & REGULAR & BIEN & BIEN \\
\hline marzo & $19 / 3 / 2017$ & Ingresos por Servicios & 85,62 & REGULAR & BIEN & BIEN \\
\hline
\end{tabular}


Tabla 3. Comparaciones múltiples por pares mediante el procedimiento de Steel-Dwass-Critchlow-Fligner.

\begin{tabular}{|l|c|c|c|c|c|}
\hline \multicolumn{1}{|c|}{ Muestra } & Frecuencia & Suma de rangos & Media de rangos & \multicolumn{2}{|c|}{ Grupos } \\
\hline BIMAS & 120 & 18891,000 & $157,425 \mathrm{z}$ & $\mathrm{A}$ & \\
\hline EXPERTO & 120 & 22553,000 & 187,942 & & $\mathrm{~B}$ \\
\hline IBIMAS & 120 & 23536,000 & 196,133 & & $\mathrm{~B}$ \\
\hline
\end{tabular}

Tabla 4. Diferencias significativas entre los métodos de evaluación empleados.

\begin{tabular}{|l|c|c|c|c|c|c|c|}
\hline Valores-p & Bimas & Experto & Ibimas & Diferencias significativas & BIMAS & EXPERTO & IBIMAS \\
\hline BIMAS & 1 & 0,046 & & & & Sí & Sí \\
\hline EXPERTO & 0,046 & 1 & 0,830 & & Sí & & No \\
\hline IBIMAS & 0,005 & 0,830 & 1 & & Sí & No & \\
\hline
\end{tabular}

Como se puede apreciar, los resultados reportan que existen diferencias significativas entre las evaluaciones obtenidas por BIMAS y las evaluaciones emitidas por expertos y obtenidas por IBIMAS. Adicionalmente, no existen diferencias significativas entre la evaluación EXPERTO e IBIMAS, comprobándose que los resultados del modelo de inferencia borroso propuesto, son más flexibles y cercanos a la realidad de los expertos en un $85 \%$ de los casos. Esto puede proporcionar a los decisores información más confiable respecto a sus organizaciones.

\section{CONCLUSIONES}

La introducción de técnicas de inteligencia artificial como la lógica difusa y los sistemas de inferencia borrosos en la evaluación de los indicadores claves de rendimiento, permitió definir un modelo de inferencia borroso para el cálculo de los indicadores claves de rendimiento en el software BIMAS. La implementación de este modelo, a partir de la definición de 12 reglas borrosas, ha propiciado una generalización parcial de algunos indicadores. Para aquellos indicadores que cuentan con un plan de cumplimiento (aquellos que fundamentalmente generan ingresos en la empresa), se ha modelado su evaluación a través de la relación existente entre el porciento de cumplimiento del plan y el tiempo de ejecución. La implementación del módulo de evaluación de indicadores basado en el sistema de inferencia borroso propuesto, acercó los resultados a los criterios de los expertos. Esto aminoró las diferencias respecto al método de evaluación existente anteriormente en el software. Aunque los resultados del modelo propuesto coinciden en un $85 \%$ de los casos con la evaluación de los expertos, queda abierta una línea de investigación que analice las reglas borrosas asociadas a la evaluación de REGULAR, debido a que en este rango fue que se encontró la mayor cantidad de no coincidencias. Otro trabajo futuro consiste en analizar qué comportamiento seguir en el caso de que el cumplimiento del indicador evaluado exceda el $100 \%$.

\section{REFERENCIAS}

[1] R.S. Kaplan and D.P. Norton."The Balanced Scorecard - Measures that Drive Performance". Harvard Business Review. 1992. Available at: https://pdfs.semanticscholar.org/6c5c/5 4be1af510a0a3b3f977b0a9d6651f3d7663. pdf. Accessed: 5 April 2018.

[2] O.P. Rud."Business intelligence success factors: tools for aligning your business in the global economy". John Wiley \& Sons. 2009. Available at: https://epdf.tips/ business-intelligence-success-factors-toolsfor-aligning-your-business-in-the-gl.html. Accessed: 5 April 2018.

[3] R. Espin. "Soft computing for business intelligence". Available at: https://books. google.com.cu/books?id=jGS5BQAAQB AJ\&pg=PA263\&lpg=PA263\&dq=lógica+ difusa+\%26+KPI\&source=bl\&ots=H7HU z75vmF\&sig=3RmKUW_6qg3iTJrtucueop 0KzWo\&hl=es\&sa=X\&ved=0ahUKEwj_9 rGL4eHYAhVCEawKHSeFD10Q6AEIPjA 


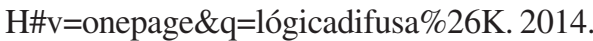
Accessed: 5 April 2018.

[4] L.A. Zadeh. "The concept of a linguistic variable and its application to approximate reasoning - III". Information Sciences. Vol. 9 $\mathrm{N}^{\circ}$ 1, pp. 43-80. 1975.

[5] B. Kosko. "Pensamiento borroso: la nueva ciencia de la lógica borrosa". Edited by 1995 Barcelona: Crítica. 1995.

[6] Y.-J. Lai and C.L. Hwang Ching-L. "Fuzzy multiple objective decision making: methods and applications". Springer-Verlag. 1994.

[7] A. Mohamed. "Design of Prediction System for Key Performance Indicators in Balanced Scorecard". International Journal of Computer Applications. Vol. 72 No 8, pp. 10-14. 2013.

[8] K. Espinal, et al. "Métrica difusa para la evaluación del desempeño en la gestión por procesos". IEC (1997) 'IEC 1131 PROGRAMMABLE CONTROLLERS', pp. 1-53. 2015.

[9] J. Sofiyabadi, B. Kolahi and C. Valmohammadi. "Key performance indicators measurement in service business: a fuzzy VIKOR approach". Total Quality Management and Business Excellence. Vol. 27 N $9-10$, pp. 1028-1042. 2016. DOI: $10.1080 / 14783363.2015 .1059272$.

[10] L. Afkham, F. Abdi and A. Rashidi. "Evaluation of service quality by using fuzzy MCDM: A case study in Iranian health-care centers". Management Science Letters. Vol. 2 $\mathrm{N}^{\circ}$ 1, pp. 291-300. 2012. DOI: $10.5267 / \mathrm{j}$. msl.2011.08.009.

[11] I. Cárdenas Tandrón and L. Sánchez Alba. "BIMAS, Solución de Gestión del Desempeño e Inteligencia Empresarial". Desoft Villa Clara, Cuba. 2015. DOI: 1471-2013.

[12] L.A. Zadeh. "“Fuzzy sets', Information and Control". Academic Press. Vol. 8 No 3, pp. 338-353. 1965. DOI: 10.1016/ S0019-9958(65)90241-X.

[13] J.A. Restrepo y J.G. Vanegas. "Internacionalización de las pymes: análisis de recursos y capacidades internas mediante lógica difusa". Revista Contaduría y Administración. Vol. 60 No 4, pp. 836-863. 2015. ISSN: 0186-1042. DOI: https://doi. org/10.1016/j.cya.2015.07.008.

[14] S.P. Perea, H. Castellanos y Y.J. Valderrama. "Estados financieros previsionales como parte integrante de un conjunto completo de estados financieros en ambiente NIIF. Una propuesta en el marco de la lógica difusa". Revista Actualidad Contable Faces. Vol. 19 No 32, pp. 113-141. 2016. ISSN: 1316-8533.

[15] Y.Y. Antelo-González y D. AlfonsoRobaina. "Análisis de la Responsabilidad Social Empresarial basado en un modelo de Lógica Difusa Compensatoria". Revista Ingeniería Industrial. Vol. $36 \mathrm{~N}^{\circ}$ 1. 2015. ISSN: 1815-5936.

[16] M.C. Lozano y F. Fuentes. "Aplicación de una metodología difusa a la negociación de la reforma laboral". Revista de Métodos Cuantitativos para la Economía y la Empresa. Vol. 16. 2013.

[17] M. León, B.L. de Lameda, C. Lameda, J.G. Chacón, M.S. Martínez, J. Rojas, J. Contreras, M. Gratenol, S. Wilches, M. Aguirre y M. Vera. "Aplicación de la lógica difusa y algoritmos genéticos para clasificación de tratamientos contra enfermedades neoplásicas malignas". Revista Archivos Venezolanos de Farmacología y Terapéutica. Vol. $35 \mathrm{~N}^{\circ} 2$, pp. 36-41. 2016. ISSN: 0798-0264.

[18] C. H. Salgado, M. Peralta, D.E. Riesco, L. Baigorria y G.A. Montejano. "Aplicación de la lógica difusa en la evaluación de modelos conceptuales de procesos de negocio". WICC. XIX Workshop de Investigadores en Ciencias de la Computación. Buenos Aires, Argentina. 2017.

[19] C.H. Salgado, M. Peralta, D.E. Riesco y G.A. Montejano. "Aplicación de la lógica difusa en la evaluación de calidad de los procesos de negocio". WICC. XVII Workshop de Investigadores en Ciencias de la Computación. Universidad Nacional de Salta, Argentina. 2015.

[20] D. Nauck and D.R. Kruse. "A neuro-fuzzy method to learn fuzzy classification rules from data". Fuzzy and Set Systems. Vol. 89 No 3, pp. 277-288. 1997. DOI: https://doi. org/10.1016/S0165-0114(97)00009-2.

[21] P. Cingolani and M.E. Bldg. "jFuzzyLogic : a Java Library to Design Fuzzy Logic Controllers According to the Standard for Fuzzy Control Programming”. International Journal of Computational Intelligence Systems. Vol. 6, pp. 61-75. 2013. DOI: https://doi.org/10.1080/18756891.2013.81 8190. 